\title{
Overexpression of NP95 mRNA by Tumor Promoters in the Promotion Phase of a Two-Stage BALB/3T3 Cell Transformation Assay
}

\author{
Ayako SAKaI, ${ }^{* a}$ Yutaka KIKUChi, ${ }^{a}$ Masashi MuroI, ${ }^{a}$ Tohru Masui, ${ }^{b}$ Chie Furihata,${ }^{c, d}$ Eriko UchidA, ${ }^{e}$ \\ Kohsuke TAKATORI, ${ }^{a}$ and Ken-ichi TANAMOTO ${ }^{f}$ \\ ${ }^{a}$ Division of Microbiology, National Institute of Health Sciences; ${ }^{b}$ Division of Genetics and Mutagenesis, National \\ Institute of Health Sciences; ${ }^{e}$ Division of Biological Chemistry and Biologicals, National Institute of Health Sciences; \\ ${ }^{f}$ Division of Food Additives, National Institute of Health Sciences; 1-18-1 Kamiyoga, Setagaya-ku, Tokyo 158-8501, \\ Japan: ${ }^{c}$ Human Genome Center, Institute of Medical Science, University of Tokyo; 4-6-1 Shirokanedai, Minato-ku, Tokyo \\ 108-8639, Japan: and ${ }^{d}$ College of Science and Engineering, Aoyama-Gakuin University; 1-1 Aoyama, Morinosato, Atsugi \\ 243-0123, Japan. Received August 13, 2002; accepted November 25, 2002
}

\begin{abstract}
We studied altered gene expressions in BALB/3T3 cells treated by different tumor promoters in the promotion phase of a transformation assay, an in vitro model of a two-stage carcinogenicity test, using fluorescent mRNA differential display analysis. Expression of the NP95 gene, which was previously found to be the gene of a murine nuclear protein associated with cell proliferation, was increased in the cultures treated by 12 - $O$-tetradecanoylphorbol-13-acetate (TPA), okadaic acid, and orthovanadate. The upregulation of NP95 mRNA was confirmed by reverse transcription-PCR, and Northern blot. TPA, okadaic acid, and orthovanadate enhanced cell proliferation as measured by a 5-bromo-2'-deoxyuridine incorporation assay. The expression level of NP95 mRNA was not affected by the treatment with typical carcinogens benzo[a]pyrene and 3-methylcholanthrene at concentrations at which they act as initiators of cell transformation. These facts may imply that the enhancement of cell transformation by these tumor promoters is due, at least in part, to the acceleration of cell proliferation. NP95 mRNA was also increased in the transformed BALB/3T3 cells. Overexpression of NP95 may also participate in the maintenance of the transformed phenotype.
\end{abstract}

Key words cell transformation; tumor promoter; gene expression; NP95; proliferation

Tumor promoters induce altered expressions of various genes in animal organs and cultured cells. However, which one(s) among the genes whose expressions are affected by tumor promoters and how they are involved in the enhancement of carcinogenesis remain to be solved. Cell transformation in vitro is regarded as a model system for carcinogenesis in vivo and has been used to study molecular and cellular mechanisms of chemical and virus carcinogenesis. ${ }^{1,2)}$ As in the mouse skin papilloma model in vivo, there are 2 stages, initiation and promotion, in the transformation of BALB/3T3 cells. ${ }^{3,4)}$ Various tumor promoters enhance the transformation of the cells initiated by a subthreshold dose of carcinogens. ${ }^{5,6}$ In this report, we examined common changes in gene expression among the cultures under treatment by 3 different tumor promoters in the promotion phase of a BALB/3T3 cell transformation assay. We postulated that it was highly possible for the common alterations of gene expression induced by different tumor promoters to be involved in enhancing cell transformation and eventually promoting carcinogenesis.

In our laboratory, numerous chemicals, including tumor promoters in vivo, have been tested for the ability to promote cell transformation using $\mathrm{BALB} / 3 \mathrm{~T} 3$ cells. 12-O-Tetradecanoylphorbol-13-acetate (TPA), okadaic acid, and orthovanadate showed potent promoting activity among the chemicals tested ${ }^{6}{ }^{6}$ TPA and okadaic acid have been reported to enhance carcinogenesis in experimental animals, ${ }^{7,8)}$ though there is no report on the promotion of carcinogenesis by orthovanadate in vivo. In this study, we treated BALB/3T3 cells with TPA, okadaic acid, or orthovanadate at concentrations that revealed an almost equal ability to enhance cell transformation for two weeks under the conditions in the promotion phase of the two-stage cell transformation assay, ${ }^{9,10)}$ screened
RNA isolated periodically from the cells using fluorescent mRNA differential display, ${ }^{11)}$ and identified an up-regulated gene, NP95.

\section{MATERIALS AND METHODS}

Cells and Culture The mouse cell line, BALB/3T3 clone A31-1-1 (JCRB0601), was provided by the Japanese Cancer Research Resource Bank, National Institute of Health Sciences (Tokyo, Japan). Transformed cells (QTTx1-1, MTx31, MTx2-3, MTTx1-1, and MO-1) were all derived from BALB/3T3 cells and obtained from transformed foci in previous experiments in our laboratory. MTx3-1 and MTx2-3 were from foci produced in the different cultures treated with MCA alone, and QTTx1-1, MTTx1-1, and MO-1 were from foci formed in the cultures treated with quercetin plus TPA, MCA plus TPA, and MCA plus okadaic acid, respectively, in two-stage transformation assays. Cells were expanded in culture, frozen in a medium containing 5\% dimethyl sulfoxide (DMSO) and $10 \%$ fetal bovine serum (FBS) and stored in liquid nitrogen as previously described. ${ }^{12)}$ Eagle's minimal essential medium containing $60 \mu \mathrm{g} / \mathrm{ml}$ kanamycin (Nissui Pharmaceutical Co., Tokyo) supplemented with $10 \%$ heatinactivated FBS (Biocell Lab., Rancho Dominguez, CA, U.S.A.) was used. Cultures were maintained in a humidified incubator with an atmosphere of $5 \% \mathrm{CO}_{2}$ in air at $37^{\circ} \mathrm{C}$.

Chemical Treatment and RNA Isolation BALB $/ 3$ T3 cells were treated with TPA (Chemicals for Cancer Research, Eden Prairie, MI, U.S.A.), okadaic acid (Wako Pure Chemical Industries, Osaka, Japan), and orthovanadate (Sigma Chemical Co., St. Louis, MO, U.S.A.) under conditions similar to the promoter treatment in the promotion phase of the two-stage transformation assay (Fig. 1). ${ }^{9,10)}$ In brief, the 


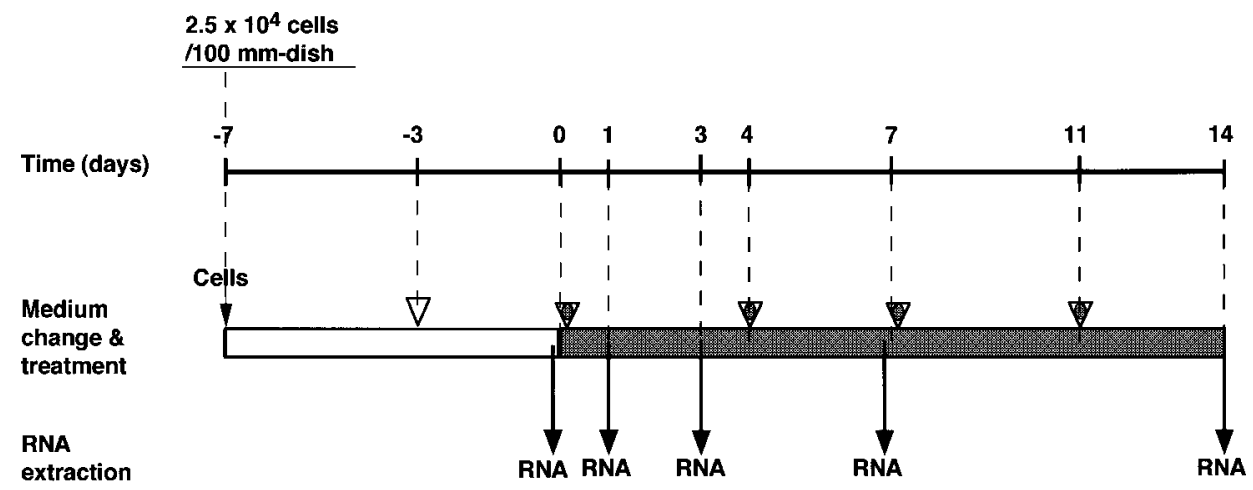

$\nabla$, Medium change; $\quad$, Medium change with the addition of a promoter

Fig. 1. Experimental Scheme of Promoter Treatment and RNA Isolation

frozen stock of BALB/3T3 cells was rapidly thawed and cultured. Actively growing cells were seeded into $100-\mathrm{mm}$ dishes containing $10 \mathrm{ml}$ of culture medium at a density of $2.5 \times 10^{4}$ cells/dish. Cells were grown in normal medium for $7 \mathrm{~d}$ to achieve subconfluence. The cultures were then treated for 2 weeks in a medium containing TPA, okadaic acid, or orthovanadate at concentrations of $200 \mathrm{ng} / \mathrm{ml}, 13 \mathrm{ng} / \mathrm{ml}$ and $1.84 \mu \mathrm{g} / \mathrm{ml}$, respectively, or a medium without any promoter (control). At these concentrations, the promoters used were approximately equally active in enhancing the focus formation in two-stage transformation assays historically in our laboratory. ${ }^{6}$ Media containing a promoter were changed twice a week as shown in Fig. 1. Total RNA was isolated from cells harvested from 1 or 2 dishes before the treatment $(0 \mathrm{~h})$ and at $24 \mathrm{~h}, 3 \mathrm{~d}, 7 \mathrm{~d}$, and $14 \mathrm{~d}$ after starting the treatment, as shown in Fig. 1, by a modified acid-guanidine thiocyanatephenol chloroform method using TRIzol Reagent (Life Technologies, Inc., Gaithersburg, MD, U.S.A.) and ISOGEN-LS (Nippon Gene, Toyama, Japan) according to the manufacturers' recommendations, stored at $-80^{\circ} \mathrm{C}$, and subjected to the mRNA differential display analysis, reverse transcriptionPCR (RT-PCR), and Northern blot analysis. The purity and integrity of the RNA isolated were determined by UV absorbance spectrophotometry and by agarose gel electrophoresis.

BALB/3T3 cells were also treated by typical carcinogens with the same procedures as the promoter-treatment mentioned above. The chemicals used were benzo[a]pyrene $(\mathrm{BaP})$ at a concentration of $50 \mathrm{ng} / \mathrm{ml}, 3$-methylcholanthrene (MCA) at concentrations of $200 \mathrm{ng} / \mathrm{ml}$ and $2 \mu \mathrm{g} / \mathrm{ml}$, and TPA at a concentration of $200 \mathrm{ng} / \mathrm{ml}$ (positive control). Total RNA was isolated before treatment $(0 \mathrm{~h})$ and at $24 \mathrm{~h}$ after starting the treatment using TRIzol and ISOGEN-LS and subjected to relative quantitative RT-PCR (RQ-RT-PCR).

Transformed BALB/3T3 cells were grown to achieve confluence and cultured further for $2 \mathrm{~d}$. Total RNA was isolated using TRIzol and ISOGEN-LS, and subjected to RQ-RTPCR.

Differential Display Analysis of mRNA Total RNA $(2.5 \mu \mathrm{g})$ isolated from the cultures treated by a promoter was reverse transcribed with $50 \mathrm{pmol} \mathrm{GT}_{15} \mathrm{VG}$ (where $\mathrm{V}$ may be $\mathrm{dG}, \mathrm{dA}$, or $\mathrm{dC}$ ) as a primer; 0.02 parts of the first cDNA strand were mixed with $10 \mathrm{pmol}$ of arbitrary $5^{\prime}$ primer (OPA-
09, 5'-GGGTAACGCC-3'; Operon Technologies, Alameda, CA, U.S.A.), 5 pmol of $\mathrm{GT}_{15} \mathrm{VG}$ and 1 unit of Taq DNA polymerase, and subjected to a low stringency PCR in a volume of $20 \mu 1 .{ }^{11)}$ PCR-amplified fragments were separated on a $5 \%$ polyacrylamide gel, stained with SYBR Green I (Molecular Probes, Eugene, OR, U.S.A.), and visualized by scanning with a fluorescence image analyzer (FluorImager 595, Molecular Dynamics, Sunnyvale, CA, U.S.A.). ${ }^{13)}$ A fragment showing unique expression $(530 \mathrm{bp})$ was cut out from the gel and reamplified by PCR.

Cloning and Sequencing The reamplified DNA fragment was cloned into the EcoRV site of pSTBlue-1 (Novagen, Madison, WI, U.S.A.), according to the manufacturer's instructions. The cloned fragment was sequenced using a Thermo Sequenase II dye terminator cycle sequencing kit (Amersham Pharmacia Biotech, Piscataway, NJ, U.S.A.) with an ABI PRISM 310 Genetic Analyzer (Perkin-Elmer, Foster City, CA, U.S.A.). A DNA sequence homology search was conducted using the BLASTN program of the National Center for Biotechnology Information (Bethesda, MD, U.S.A.).

RT-PCR with Specific Primers and Northern Blot Analysis PCR was carried out at high stringency with specific primers, which were nested in the open reading frame of the NP95 gene, and a first-strand cDNA set different from that used for the differential display. The specific oligonucleotide primers were $5^{\prime}$-GCAAGGATGATGAAAACAAACC-3' (5' primer) and 5'-CCAGCCAGGACCAATGAGTAGG-3' (3' primer). The PCR products were examined on a $3 \%$ agarose gel.

For Northern blot analysis, $10 \mu \mathrm{g}$ of total RNA was resolved on a $1.2 \%$ agarose $-0.66 \mathrm{~m}$ formaldehyde gel, blotted onto a Biodyne Nylon Membrane (Pall BioSupport Division, Glen Cove, NY, U.S.A.), and then hybridized with a ${ }^{32} \mathrm{P}-\mathrm{la}-$ beled probe which was prepared by EcoRI digestion from the plasmid used for sequencing. Washed membranes were exposed on an imaging plate and analyzed by BAS 1500 (Fuji Film, Tokyo, Japan). Before being blotted onto the membrane, RNAs on the agarose gel were stained with SYBR Green II (Molecular Probes, Eugene, OR, U.S.A.) and visualized by the fluorescence image analyzer to confirm the quality and that the amounts of RNAs were equal.

RQ-RT-PCR Total RNAs isolated from the cultures treated with typical carcinogens and transformed cells were 
reverse transcribed with random primers $\left(\mathrm{N}_{6}\right)$; RQ-RT-PCR was performed with the reverse transcripts, the specific primers, and Quantum RNA Alternate 18S Internal Standards (Ambion, Austin, Tex, U.S.A.), according to the manufacturer's recommendations. The PCR products were examined on a $3 \%$ agarose gel.

5-Bromo-2'-deoxyuridine (BrdU) Incorporation Assay BALB/3T3 cells (250/well) were inoculated on a 96-well plate and grown for $7 \mathrm{~d}$ in normal medium, and the culture was refilled with an exhausted medium, a fresh normal medium, or a medium containing TPA, okadaic acid, or orthovanadate at the same concentrations as in "Chemical Treatment and RNA Isolation". The cells were labeled with BrdU for $1 \mathrm{~h}$ from $24 \mathrm{~h}$ after the start of the chemical treatment. The BrdU incorporated by the cells was measured using a Cell Proliferation ELISA, BrdU (colorimetric) kit (Roche Diagnostics, Mannheim, Germany) according to the manufacturer's instructions.

\section{RESULTS}

Differential Expression of NP95 mRNA BALB/3T3 cells were treated with TPA, okadaic acid, or orthovanadate under the conditions in the promotion phase of the two-stage cell transformation assay. The total RNAs were isolated according to the schedule summarized in Fig. 1 and subjected to mRNA differential display analysis. The analysis revealed that the densities of the bands at $530 \mathrm{bp}$ were different between tumor promoter-treated cultures and the control at $24 \mathrm{~h}$ after the start of treatment and changed with the elapse of treatment time (Fig. 2a). The $530 \mathrm{bp}$ fragment was cloned into the pSTBlue-1 cloning vector and sequenced. The sequence was entirely identical to 2198-2710 of NP95 cDNA (GenBank accession number D87908).

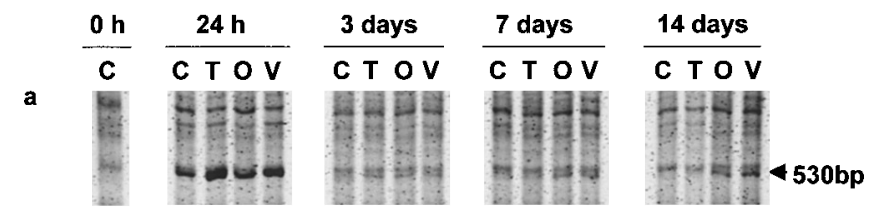

b
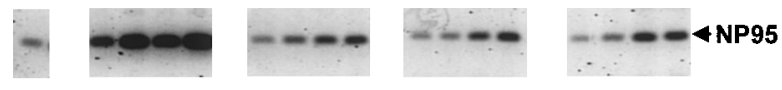

C
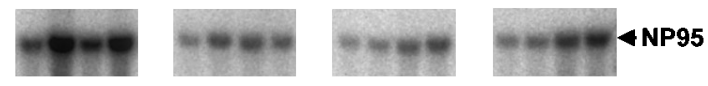

d

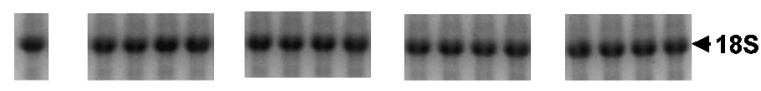

Fig. 2. Changes in Expression of NP95 mRNA in BALB/3T3 Cells under Tumor Promoter Treatment

Cells were grown in 100-mm dishes to achieve subconfluence, and the cultures were treated in a fresh medium without any promoter (control, C), or a medium containing TPA $(200 \mathrm{ng} / \mathrm{ml}, \mathrm{T})$, okadaic acid $(13 \mathrm{ng} / \mathrm{ml}, \mathrm{O})$, or orthovanadate $(1.84 \mu \mathrm{g} / \mathrm{ml}, \mathrm{V})$. Total RNA was isolated before the treatment $(0 \mathrm{~h})$, at $24 \mathrm{~h}, 3 \mathrm{~d}, 7 \mathrm{~d}$, and $14 \mathrm{~d}$ after starting the treatment as summarized in Fig. 1 and subjected to mRNA differential display analysis (a), RT-PCR with specific primers (b), and Northern blot analysis (c) as detailed in Materials and Methods. SYBR Green II-staining of 18S rRNA (d) is presented to confirm equal amounts of total RNA.
RT-PCR with specific primers that were settled in the open reading frame of the NP95 gene and Northern blot analysis were carried out. The expression behavior of NP95 mRNA was confirmed to be similar in all differential display analysis, RT-PCR with specific primers and Northern blot analysis (Fig. 2). At $24 \mathrm{~h}$ after the start of treatment, the expression of NP95 mRNA was increased in the control cultures which were treated with a fresh medium instead of promoter-containing medium. In promoter-treated cultures, the expression of NP95 mRNA at $24 \mathrm{~h}$ was elevated more than the increased level in the control. Although the NP95 expression elevated by fresh medium alone declined to the normal level within $3 \mathrm{~d}$, the promoter-treated cells at $3 \mathrm{~d}$ retained higher NP95 expression than the control cells. The increased expression of NP95 mRNA was also clear at 7 and $14 \mathrm{~d}$ in the cultures treated with okadaic acid and orthovanadate, though it was obscure in TPA-treated cultures.

Change in the Activity of Cell Proliferation NP95 was previously found to be the gene of a murine nuclear protein associated with proliferation; ${ }^{14)}$ therefore, we examined the changes in DNA synthesis following treatment with TPA, okadaic acid, and orthovanadate using a BrdU incorporation assay. Fresh medium markedly activated cell proliferation at $24 \mathrm{~h}$ after the replacement of medium, and TPA, okadaic acid, and orthovanadate added to the fresh medium further enhanced cell proliferation induced by fresh medium (Fig. $3)$.

Change in NP95 mRNA Expression by Typical Carcinogens In order to determine whether the overexpression of NP95 at $24 \mathrm{~h}$ of promoter treatment was unique to tumor promoters, BALB/3T3 cells were treated for $24 \mathrm{~h}$ with typical carcinogens at two levels of concentrations, at which they act as initiators $(\mathrm{BaP}$ at a concentration of $50 \mathrm{ng} / \mathrm{ml}$ and MCA at a concentration of $200 \mathrm{ng} / \mathrm{ml}$ ) and as a complete carcinogen (MCA at a concentration of $2 \mu \mathrm{g} / \mathrm{ml}$ ) in the BALB/3T3 cell transformation assay., ${ }^{4,6,10)}$ The expression of NP95 mRNA was compared using RQ-RT-PCR. As shown in Fig. 4, the degree of increase in NP95 expression of cells treated with medium containing $\mathrm{BaP}$ at the concentration of $50 \mathrm{ng} / \mathrm{ml}$ and MCA at the concentration of $200 \mathrm{ng} / \mathrm{ml}$ was similar to that of the control cells treated with fresh medium without a chemical. However, the expression level of NP95

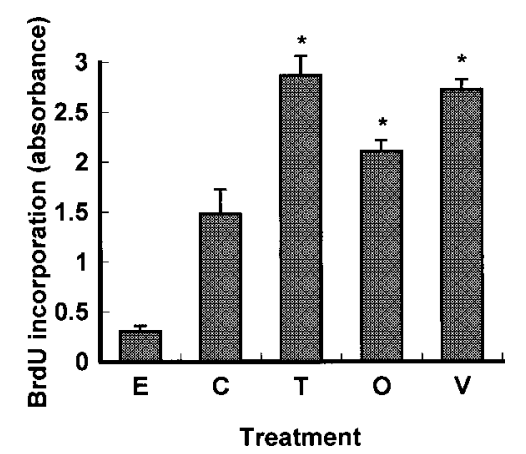

Fig. 3. Incorporation of BrdU into BALB/3T3 Cells Treated with Tumor Promoters

Cells were grown in a 96-well plate to achieve subconfluence, and treated for $24 \mathrm{~h}$ in exhausted medium (E), fresh medium (control, C) or medium containing TPA (T), okadaic acid (O), or orthovanadate (V) at the same concentrations as in Fig. 2. Cultures were then labeled with BrdU for an hour. Values represent the averages of 8 wells. Bars, S.D.; $*, p<0.05$ versus $\mathrm{C}$. 


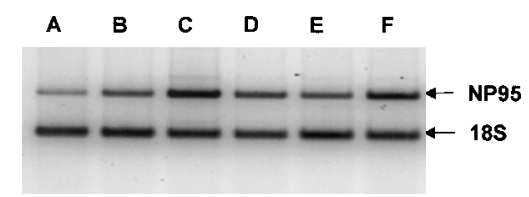

Fig. 4. Changes in Expression of NP95 mRNA in BALB/3T3 Cells after Treatment with Typical Carcinogens

Cells were grown in 100-mm dishes to achieve subconfluence, and the cultures were treated in a fresh medium without any promoter (control, B), or a medium containing TPA $(200 \mathrm{ng} / \mathrm{ml}, \mathrm{C}), \mathrm{BaP}(50 \mathrm{ng} / \mathrm{ml}, \mathrm{D})$, or MCA $(200 \mathrm{ng} / \mathrm{ml}$ and $2 \mu \mathrm{g} / \mathrm{ml}, \mathrm{E}$ and F, respectively). Total RNA was isolated before the treatment (A) and at $24 \mathrm{~h}(\mathrm{~B}-\mathrm{F})$ after starting the treatment and then subjected to RQ-RT-PCR. The PCR products were resolved on $3 \%$ agarose gel and stained with SYBR Green I.

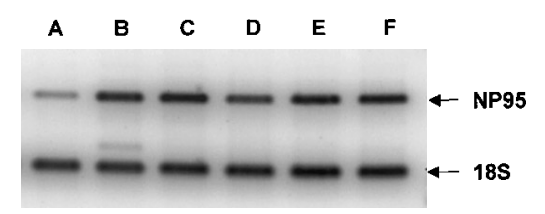

Fig. 5. Overexpression of NP95 mRNA in Transformed BALB/3T3 Cells

RQ-RT-PCR products of RNA isolated from nontransformed and transformed BALB/3T3 cells were resolved on 3\% agarose gel and stained with SYBR Green I. A, normal cells; B-F, transformed cells in the order of QTTx1-1, MTx3-1, MTx2-3, MTTx1-1, and MO-1.

in the cultures treated by MCA at the concentration of $2 \mu \mathrm{g} / \mathrm{ml}$ was higher than the level in the control cultures.

Overexpression of NP95 mRNA in Transformed Cells The expression of NP95 mRNA was compared between transformed and nontransformed BALB-3T3 cells using RQRT-PCR with specific primers. The expression of NP95 mRNA was increased in all transformed cells examined (Fig. 5).

\section{DISCUSSION}

TPA, okadaic acid, and orthovanadate, all of which act as promoters of cell transformation, were shown to elevate the expression of NP95 mRNA in the promotion phase of a BALB/3T3 cell transformation assay. In the early period of promoter treatment, the expression of NP95 mRNA was elevated or enhanced in all the cultures treated with these promoters, and in the later period, the increased expression was maintained in the cultures treated with okadaic acid and orthovanadate. NP95 was previously identified as a 95-kDa mouse nuclear protein associated with cell proliferation; ${ }^{15}$ the cDNA encodes a protein with an open reading frame containing 782 amino acids. NP95 contains a potential ATP/GTP binding site, a zinc finger motif, a putative cyclin $\mathrm{A} / \mathrm{E}$ cdk2 phosphorylation site, and a retinoblastoma binding motif. ${ }^{14}$ ) Although its physiological functions are as yet unclear, the expression is observed mostly in proliferating cells. ${ }^{16)}$

In this study, the expression of NP95 mRNA in the control cultures was markedly increased at $24 \mathrm{~h}$ after the beginning of treatment with fresh medium containing no promoter. It is reported that the levels of NP95 mRNA and protein were extremely low in quiescent cells and stimulation with $10 \%$ serum increased their expressions. ${ }^{16)}$ We confirmed by RTPCR that the increase in NP95 mRNA at $24 \mathrm{~h}$ was mainly due to the serum which fresh medium contained. That is, NP95 mRNA was increased in the cultures whose medium was replaced with the exhausted medium supplemented with fresh serum but not with exhausted medium only (data not shown). TPA, okadaic acid, and orthovanadate further enhanced the NP95 expression increased by fresh medium. The enhancement was larger with TPA and orthovanadate than with okadaic acid. NP95 mRNA increased by fresh medium returned to a normal level within $3 \mathrm{~d}$. Because all RNA samples, except those at $24 \mathrm{~h}$, were isolated at $3 \mathrm{~d}$ after the replacement of medium with/without a promoter as shown in Fig. 1, NP95 mRNA levels exhibited in Fig. 2, except those at $24 \mathrm{~h}$, were considered not to be affected by medium change.

At $24 \mathrm{~h}$ after the beginning of treatment, the activity of DNA synthesis was increased in the control cells treated with fresh medium only, just as noted in previous reports where DNA synthesis and cell division were induced by serum and medium change in BALB/3T3 cells. ${ }^{17,18)}$ The activity of DNA synthesis was further accelerated in the cells treated with promoters. The degree of acceleration was larger in the cultures treated with TPA and orthovanadate than in the cultures treated with okadaic acid. Thus, the change in the activity of DNA synthesis was very similar to the alteration in the expression of NP95 mRNA at $24 \mathrm{~h}$ after the start of treatment, suggesting that the increased expression of NP95 mRNA is associated with cell proliferation in the promotion phase of the two-stage BALB/3T3 cell transformation assay.

In the middle and later periods of promoter treatment, the effects of promoters on the expression of NP95 were different among the promoters examined. At 7 and $14 \mathrm{~d}$, the expression was elevated in the cultures exposed to okadaic acid and orthovanadate but not in the cultures exposed to TPA. The expression might be elevated by TPA at moments different than that when RNA was isolated in this experiment. The interval of $3 \mathrm{~d}$ might be too long to trace RNA expression. An experiment where RNAs are isolated at short intervals during tumor promoter treatment has been planned and the results will be published elsewhere. TPA and orthovanadate increased and okadaic acid decreased the cell density after $3 \mathrm{~d}$ of treatment, as described in previous papers; ${ }^{9,10,17)}$ these effects on cell density caused great differences in cell numbers in a well of the 96 well plate among the treatment groups after $3 \mathrm{~d}$. Dispersion of cell numbers among wells within the same treatment group was also large after $3 \mathrm{~d}$ of treatment. These factors interfered with the analysis using a BrdU incorporation assay to demonstrate the relationship between NP95 expression and cell proliferation activity in the middle and later periods of promoter treatment (data not shown).

In this study, $\mathrm{BaP}$ at a concentration of $50 \mathrm{ng} / \mathrm{ml}$ and $\mathrm{MCA}$ at a concentration of $200 \mathrm{ng} / \mathrm{ml}$ did not enhance the increase in NP95 expression induced by fresh normal medium, whereas MCA at a concentration of $2 \mu \mathrm{g} / \mathrm{ml} \mathrm{did}$. This fact suggests that the enhancement of NP95 expression is not specific to tumor promoters and is caused by typical carcinogens at a sufficient concentration. According to the theory of two-stage carcinogenesis, it is generally considered that both initiating and promoting activities are required for accomplishment of chemical carcinogenesis; a tumor promoter has promoting activity but not initiating activity, while a complete carcinogen has both activities. BaP at the concentration of $50 \mathrm{ng} / \mathrm{ml}$ and MCA at the concentration of $200 \mathrm{ng} / \mathrm{ml}$ act only as initiators for BALB/3T3 cell transformation and always achieve a 
statistically significant transformation of the cells in the presence of TPA or another promoter but not in the absence of a promoter in our laboratory. MCA at a concentration of $2 \mu \mathrm{g} / \mathrm{ml}$ is able to complete transformation of BALB/3T3 cells without a tumor promoter, indicating that MCA at $2 \mu \mathrm{g} / \mathrm{ml}$ has both initiating and promoting activities. The promoting activity of MCA at $2 \mu \mathrm{g} / \mathrm{ml}$ might cause enhancement of NP95 expression, though further studies using more typical carcinogens are necessary to confirm this hypothesis.

In this study, all the transformed BALB/3T3 cells examined clearly showed overexpression of NP95 mRNA compared to normal BALB/3T3 cells. The transformed cells examined were derived from foci produced in the different cultures exposed to various chemicals. It has been reported that the NP95 protein is up-regulated in all mouse lymphoma cell lines. ${ }^{15)}$ Overexpression of NP95 may participate in the maintenance of the transformed phenotype.

Our results did not demonstrate that the elevated expression of NP95 plays a specific role in the enhancement of transformation caused by tumor promoters. However, the facts that TPA, okadaic acid, and orthovanadate induced overexpression of mRNA of NP95, a gene of a nuclear protein associated with proliferation, and acceleration of DNA synthesis in the promotion phase of BALB/3T3 cell transformation may imply that the enhancement of cell transformation by these tumor promoters is due, at least in part, to the activated cell proliferation. Increased expression of NP95 mRNA in BALB/3T3 cells after treatment by a chemical may serve as one of the indexes to identify a tumor promoter.

Acknowledgment This study was supported in part by Grants-in-Aid for Scientific Research (C) (10672118 and 14572120) from the Japan Society for the Promotion of Science to Dr. A. Sakai.

\section{REFERENCES}

1) Barrett J. C., Kakunaga T., Kuroki T., Neubert D., Trosko J. E., Vasiliev J. M., Williams G. M., Yamasaki H., "Long-Term and ShortTerm Assays for Carcinogens: A Critical Appraisal," IARC Scientific Publication no. 83, ed. by Montesano R., Bartsch H., Vainio H., Wilbourn J., Yamasaki H., IARC, Lyons, 1986, pp. 267-286.

2) Nagalakshmi K., Mutation Res., 447, 281-286 (2000).

3) Hirakawa T., Kakunaga T., Fujiki H., Sugimura T., Science, 216, 527-529 (1982).

4) Sakai A., Sato M., Mutation Res., 214, 285-296 (1989).

5) Kuroki T., Sasaki K., "Transformation Assay of Established Cell Lines: Mechanisms and Application," IARC Scientific Publication no. 67, ed. by Kakunaga T., Yamasaki H., IARC, Lyons, 1985, pp. 93118.

6) Sakai A., Iwase Y., Nakamura Y., Sasaki K., Tanaka N., Umeda M., ATLA, 30, 33-59 (2002).

7) Berenblum I., Cancer Res., 1, $44-48$ (1941).

8) Sugamuma M., Fujiki H., Suguri H., Yoshizawa S., Hirota M., Nakayasu M., Ojika M., Wakamatsu K., Yamada K. Sugimura T., Proc. Natl. Acad. Sci. U.S.A., 85, 1768-1771 (1988).

9) Sakai A., Fujiki H., Jpn. J. Cancer Res., 82, 518-523 (1991).

10) Sakai A., Carcinogenesis, 18, 1395-1399 (1997).

11) Ito T., Kito K., Adachi N., Mitsui Y., Hagiwara H., Sakaki Y., FEBS Lett., 351, 231-236 (1994).

12) Sakai A., Mutation Res., 493, 161-166 (2001).

13) Furihata C., Oka M., Yamamoto M., Ito T., Ichinose M., Miki K., Tatematsu M., Sasaki Y., Reske K., Cancer Res., 57, 1416-1418 (1997).

14) Fujimori A., Matsuda Y., Takemoto Y., Hashimoto Y., Kubo E., Araki R., Fukumura R., Mita K., Tatsumi K., Muto M., Mammalian Genome, 9, 1032-1035 (1998).

15) Muto M., Utsuyama M., Horiguchi T., Kubo E., Sado T., Hirokawa K., Cell Prolif., 28, 645-657 (1995).

16) Miura M., Watanabe H., Sasaki T., Tatsumi K., Muto M., Experimental Cell Research, 263, 202-208 (2001).

17) Sakai A., Miyata N., Takahashi A., Cancer Lett., 115, 213-220 (1997).

18) Sakamoto Y., Takeda Y., Takagi H., Tsuchiya T., Shoji A., Miyazaki K., Umeda M., Cancer Lett., 136, 159-165 (1999). 